\title{
A Rare Cause of Reversible Unilateral Breast Swelling: A Case Report
}

\author{
Farhad Fakhrudin Vasanwala ${ }^{\mathrm{a}, \mathrm{c}}$, Yuen Li NG ${ }^{\mathrm{b}}$
}

\begin{abstract}
We report a rare case of unilateral breast swelling due to congestive heart failure. Our patient was initially suspected to have carcinoma of the breast based on clinical findings. However, imaging studies showed considerable oedema in the skin, subcutaneous and glandular tissues of the right breast, without a mass lesion. The oedema resolved with diuretic treatment. This case illustrates the point to consider congestive heart failure as a cause of unilateral breast swelling especially in a patient with a background of congestive heart failure and hypoalbuminaemia.
\end{abstract}

Keywords: Unilateral breast swelling; Inflammatory carcinoma of the breast; Congestive heart failure; Hypoalbuminaemia

\section{Introduction}

With our aging population, congestive heart failure and breast malignancies are increasingly more prevalent among the elderly worldwide. Besides knowing the textbook presentation of these diseases, familiarizing with the atypical presentations of these common diseases are also important. This awareness would facilitate early diagnosis and management, reducing unnecessary investigations and patient morbidity.

We report a rare case of unilateral breast swelling in an elderly lady with a background of congestive heart failure.

\footnotetext{
Manuscript accepted for publication November 16, 2012

a Department of Family Medicine and Continuing Care, Singapore General Hospital, Outram Road, Singapore

${ }^{\mathrm{b}}$ Department of Diagnostic Radiology, Singapore General Hospital, Outram Road, Singapore

${ }^{\mathrm{c} C}$ Corresponding author: Farhad Fakhrudin Vasanwala, Department of Family Medicine and Continuing Care, Singapore General Hospital, Outram Road, Singapore

Email: farhad.fakhrudin.vasanwala@sgh.com.sg
}

doi: http://dx.doi.org/10.4021/jmc978w

\section{Case Report}

Madam P, a 72-year-old lady homemaker premorbidly ADL independent presented with shortness of breath, progressive right breast and right upper limb swelling for the past two weeks. She had increased productive sputum, but no fever, and no rhinorrhoea. She denied any history of trauma to the breast. She had a past medical history of diabetes, congestive heart failure, valvular heart disease (EF 25\%) with severe aortic stenosis, moderate severe mitral regurgitation, severe tricuspid regurgitation, and regional wall motion abnormality. She also had gout, iron and Vitamin B12 deficient anaemia, nephropathy with hypoalbuminaemia and was on follow up with the cardiologist, rheumatologist and endocrinologist for her chronic medical problems.

Patient was alert, afebrile, tachypnoeic with respiratory rate of $24 / \mathrm{min}$, heart rate of $63 / \mathrm{min}$ regular rhythm, blood pressure of 120/60 mmHg. Heart sounds were normal with a systolic murmur and JVP was elevated. There were bilateral crepitations of the lungs; right lung was more than the left. Abdomen was soft, non tender with no organomegaly. There were bilateral lower limb pitting oedema up to the knees. Clinical breast examination showed an enlarged right breast

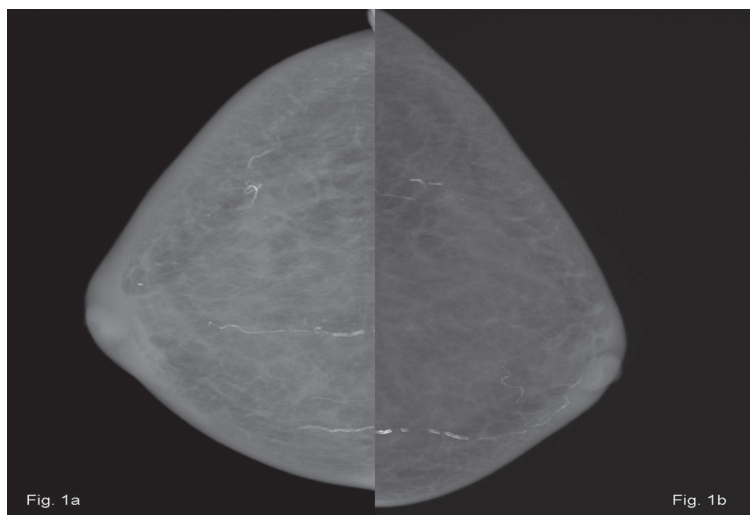

Figure 1. Craniocaudal mammograms of the right breast (a) showing enlargement with oedema, relative to the left breast (b). 


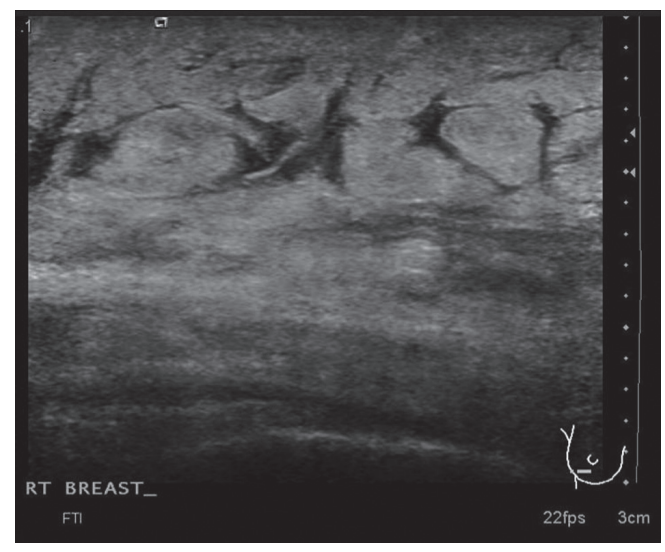

Figure 2. Ultrasound image of the right breast showing significant oedema of the glandular tissue.

mainly around the nipple-areolar area with diffusely thickened peau d'orange skin. There was no pitting oedema of the breast or palpable mass. No nipple discharge, retraction nor lymphadenopathy was detected. The right upper limb oedema was more pronounced than the left.

The provisional diagnoses were congestive heart failure with concomitant inflammatory carcinoma of the breast. Patient was started on the standard management for heart failure, for example, furosemide, spironolactone, potassium supplementation, beta blockers, ACE (angiotensin-converting-enzyme inhibitor) inhibitors with fluid and salt restriction. Mammography of both breasts and ultrasound of the right breast were performed in view of the suspected neoplastic pathology.

The digital mammograms (Fig. 1) showed an enlarged right breast. There was breast oedema bilaterally, worse on the right. The skin and areola of the right breast were thicker. Curvilinear vascular calcifications were also present in the breasts. Ultrasound of the right breast (Fig. 2) confirmed considerable oedema in the skin, subcutaneous and glandular tissues of the right breast. No mass lesion was seen.

After 2 days the right breast swelling resolved. Patient declined further cardiac intervention and was stable on discharged one week after admission with follow-up outpatient appointment.

\section{Discussion}

Our initial impression of the unilateral breast swelling was an inflammatory breast carcinoma. This is a rare, highly aggressive form of primary breast cancer that comprises 1 to $6 \%$ of all the cases of breast cancer. The following signs and symptoms suggested the diagnosis: 1). The rapid onset of oedema (swelling), and a peau d'orange appearance, without a lump that can be felt [1];2). The symptoms have been present for less than 6 months [1].
However, there was no increase in warmth or erythema present.

Breast oedema may present in either unilateral or bilateral manner. Bilateral breast oedema may occur in patients with systemic illnesses like renal failure, heart failure, and liver disease [2]. Oedema in breast carcinoma may be due to congestion or tumor cell infiltration of the dermal and the intramammary lymphatics, whereas unilateral breast oedema in congestive heart failure may be caused by patient's tendency to lie on one side leading to dependent oedema [3]. Our patient was lying predominantly on the right side which accounted for the right unilateral breast oedema.

Congestive heart failure is a rare cause of unilateral breast oedema, which should resolve when the underlying condition is appropriately treated. Our patient's breast oedema resolved after 2 days of diuretic therapy. Her breast swelling would also have been aggravated by her hypoalbuminaemia secondary to her diabetic nephropathy [4].

Other differential diagnoses include lymphatic obstruction, mastitis, lymphoma, and post radiation changes [4]. However, she did not have a prior history of radiotherapy, and lymphoma was very unlikely since the swelling resolved with diuretic therapy. Mastitis in an elderly non-lactating lady is highly unlikely.

Although breast congestion due to heart failure has been first described by Stolz et al in 1974, even now it has not been described as a cause of breast swelling in standard authoritative medical textbooks $[5,6]$.

Thus, worldwide where congestive heart failure is becoming increasingly more prevalent, there is a need to be familiar with its atypical presentations. This awareness would facilitate early diagnosis and management, reducing unnecessary investigations and patient morbidity.

\section{References}

1. Drenthen W, Hoendermis ES, Moons P, Heida KY, RoosHesselink JW, Mulder BJ, Van Dijk AP, et al. Menstrual cycle and its disorders in women with congenital heart disease. Congenit Heart Dis. 2008;3(4):277-283.

2. McElligott G, Harrington MG. Heart failure and breast enlargement suggesting cancer. Br Med J (Clin Res Ed). 1986;292(6518):446.

3. Jung JH, Min PK, Moon YW, Shim WH. Congestive heart failure and a swollen breast. Lancet. 2004;363(9404):210.

4. An YY, Kim SH, Cha ES, Kim HS, Kang BJ, Park CS, Jung NY, et al. Diffuse infiltrative lesion of the breast: clinical and radiologic features. Korean $\mathrm{J}$ Radiol. 2011;12(1):113-121.

5. Stolz JL, Friedman AK, Arger PH. Breast carcinoma simulation. Mammography in congestive heart failure mimics acute mastitis and advanced carcinoma. JAMA. 
1974;229(6):682-683.

6. Longo D, Fauci A, Kasper D, Hauser S, Jameson J,
Loscalzo J. Harrison's Principles of Internal Medicine, 18th ed, USA: McGraw Hill Professional, 2011. 